\title{
Estado de la gestión municipal de los residuos sólidos en la provincia de Cartago
}

\author{
Municipal solid waste management in Cartago province
}

Silvia M. Soto-Córdoba'

Lilliana Gaviria-Montoya ${ }^{2}$

Fecha de recepción: 6 de agosto del 2013 Fecha de aprobación: 24 de setiembre del 2013

Soto-Códoba, S; Gaviria-Montoya, L. Estado de la gestión municipal de los residuos sólidos en la provincia de Cartago. Tecnología en Marcha. Número Especial Pág 105-1 17.

I Catedrática, Ingeniería Ambiental, Instituto Tecnológico de Costa Rica. Correo electrónico: ssoto@itcr.ac.cr, tel. (506)-2550-9294

2 Catedrática, Ingeniería Ambiental, Instituto Tecnológico de Costa Rica. Correo electrónico: Igaviria@itcr.ac.cr, tel. (506)-2550-9/38 


\section{Silvia M. Soto Córdoba}

Profesora Catedrática con 24 años de laborar en el Instituto Tecnológico de Costa Rica (ITCR), ha impartido cursos en la UCR, UNA y UNED en diversos temas que incluyen química básica 1 y 2 , química orgánica, química analítica, manejo de residuos sólidos, protección ambiental, química verde, a nivel de grado, maestría y doctorado.

Desarrolló sus estudios doctorales en Chile, en la Universidad de Concepción donde obtuvo una nota de presentación de tesis doctoral de 100. Su proyecto estudió la degradación de compuestos orgánoclorados utilizando bacterias nativas extraídas desde suelos contaminados.

Fue coordinadora del Centro de investigación en protección ambiental del ITCR, coordinadora del proyecto de subvención de la Federación de Municipalidades de Cartago, financiado por la comu- nidad económica europea, en el tema de mejorar la gestión ambiental municipal en el tema de los residuos sólidos, ha participado en charlas comunales y programas de recuperación de residuos sólidos.

Lleva 10 años escribiendo ponencias para el programa estado de la nación en el tema de residuos sólidos en el país.

Actualmente es profesora de la carrera de ingeniería ambiental del ITCR, de la maestría en recursos renovables de la UNED, y es directora de tesis de maestría en recursos naturales y doctorado en ciencias para el desarrollo.

Coordinó los trabajos finales de graduación y el comité técnico de la escuela de química.

Coordinadora nacional de la red REDCICLA.

Es madre de tres hijos, casada y vive en Zapote. 


\section{Lilliana Gaviria Montoya}

Para Lilliana Gaviria Montoya, ingeniera química con 20 años de trabajar en el Instituto Tecnológico de Costa Rica (ITCR), la investigación debe desarrollarse en la Universidad, "el lugar idóneo donde deben gestarse las soluciones a los problemas de la sociedad costarricense entendiéndose: industria, bienestar social, economía, etc.".

Lilliana Gaviria es especialista en ingeniería sanitaria, graduada en el Instituto Internacional de Ingeniería, Hidráulica y Ambiente, de Holanda, y tiene una maestría en administración de empresas del ITCR.

Esa formación no la obtuvo por casualidad: desde hace muchos años, Lilliana ha mostrado preocupación por el ambiente, y prueba de ello es la tesis que presentó en 1986 para graduarse como licenciada en Ingeniería Química, sobre el tema del tratamiento de aguas residuales. Esta investigadora cree firmemente que "si no protegemos el ambiente vamos a terminar en un mundo sin calidad de vida y aunque tengamos mucho dinero no tendremos qué, comprar paradójicamente hablando".

Lilliana Gaviria llegó a trabajar alTEC en 1989, como profesora de la Escuela de Química. Anteriormente había laborado en la empresa privada y cuenta que se sentía desilusionada por la forma discriminatoria en que se trataba a los empleados de puestos operativos. Por eso realizó el cambio.

Desde entonces, esta profesora e investigadora ha ocupado diversos cargos, entre ellos el de coordinadora del Centro de Investigación en Protección Ambiental (CIPA) y el de coordinadora de la carrera de Ingeniería Ambiental; este último cargo lo ejerce desde el año 2006 y hasta la fecha.

Lilliana también ha sido consultora del Centro Nacional de Producción más Limpia desde su creación en 1995, e instructora de numerosos cursos de capacitación dirigidos a comunidades, empresas privadas, grupos organizados y municipalidades.

Además, ha sido representante del TEC ante otras organizaciones públicas y privadas, ha participado en seminarios y congresos dentro y fuera del país, y ha escrito numerosos artículos en el campo de su especialidad.
Esta investigadora ha participado como coordinadora en varios proyectos de investigación, tales como: Medidas de remediación en parques costeros (2007); Parque Ambiental Municipal (2007); Proyecto de Gestión Ambiental Municipal del Cantón Central de Cartago (2004-2006); Introducción de la dimensión ambiental en el currícula del Instituto Tecnológico de Costa Rica (2004-2006); e Implementación de producción más limpia en industria de plástico de Costa Rica (2002).

Junto con la máster Hilda Quesada, creó la licenciatura en Ingeniería Ambiental del Instituto Tecnológico de Costa Rica, en el año 2005.

Para transferir los conocimientos que genera por medio de sus proyectos de investigación, Lilliana Gaviria afirma que ella y el grupo del Centro de Investigación en Protección Ambiental (CIPA), en el que participa, ofrecen capacitación a quien se le está ofreciendo la solución y dan seguimiento a la implementación de las actividades del proyecto en cuestión. Piensa que la transferencia de tecnología tiene muchas aristas $y$, a veces, en los proyectos de investigación no se tiene el tiempo suficiente para hacer una adecuada transferencia de conocimientos, especialmente por el concepto de investigación y los recursos con que cuenta la VIE.

Lilliana Gaviria nació en Aguas Zarcas de San Carlos, en un hogar de inmigrantes colombianos. Tiene dos hermanas y un hermano, nacidos todos en Colombia. Ella, la menor de la familia, "vino a nacer en este maravilloso país".

Disfruta mucho de los bosques, por lo que tiene entre sus pasatiempos caminar durante varias horas en estos y por distintos lugares del país. También le gusta la lectura, especialmente la que tiene que ver con el campo del esoterismo, los extraterrestres y los animales. Le gusta el buen cine, pero para apreciarlo en una sala de cine, no en DVD.

Lilliana también es adicta al ejercicio y lo practica en sus ratos libres, que también comparte con su gatita consentida llamada Tiny. 


\section{Palabras clave}

Residuos sólidos; Gestión Municipal; GIRS.

\section{Resumen}

Este artículo resume una parte de los resultados obtenidos mediante el proyecto de subvención EUROPEAID/I26635/M/ACT/CR, otorgado a la FUNDATEC y cuyo beneficiario es la Federación de Municipalidades de Cartago, Costa Rica. El proyecto contó con un financiamiento de 74,920 euros e incluyó todas las municipalidades y concejos municipales de distrito de la provincia de Cartago. Además, se incorporan los resultados de las entrevistas de campo, visitas a centros de acopio, visitas a las municipalidades durante los años 20I0, 20II y 2012 y revisión de literatura.

Se describe el estado actual de la gestión de los residuos sólidos en Cartago, se incorporan los datos de generación y se identifican los principales sitios de disposición, las empresas recicladoras y los principales problemas.

Se espera que la información presentada aquí sirva de base para la futura construcción de planes de gestión de residuos sólidos municipales y la capacitación de organizaciones sociales y comunales de la provincia de Cartago.

\section{Keywords}

Solid Waste; Municipal environmental administration; Integrated management of solid waste.

\section{Abstract}

This paper resumes the principals results obtained by the grant EUROPEAID/I26635/M/ACT/CR", that was realized by FUNDATEC, and whose beneficiary was the "Federación de Municipalidades de Cartago, Costa Rica", the Project received a funding of 74,920 euros. We work with all the Municipalities of the Cartago Province. In addition, we show the results of the interviews of social actors, visits to the recycle sites, visits of municipalities, during the years 2010,2011 and 2012, and the review of literature.

We describe the actual situation of the management of solid waste in Cartago, determinate the generation rates by person and identified the principal landfill disposes, the recycle companies and determinate the main problems associated with the solid waste.

It is hope that the information presented here, provides the basis for the future construction of plans of municipal solid waste management, and for the capacitation of community organization in the province of Cartago. 


\section{Introducción}

Durante 2010, diez profesores de la Escuela de Química del Instituto Tecnológico de Costa Rica (ITCR), el Centro de Investigación en Protección Ambiental y la Escuela de Administración de Empresas, junto con cuatro colaboradores externos, desarrollaron el proyecto de subvención EUROPEAID/I 26635/M/ACT/CR, destinado a apoyar la gestión integral de los residuos sólidos en las municipalidades y concejos municipales de distrito de la provincia de Cartago.

Los beneficiarios directos capacitados en el proyecto ascendieron a 496 hombres y 986 mujeres, incluyendo funcionarios municipales de los cantones de Alvarado, Jiménez, Turrialba, Tucurrique, Cervantes, Cartago, La Unión y Paraíso y dos de la municipalidad de Matina. También se capacitaron grupos comunales organizados para el manejo de los residuos sólidos de Cartago (San Nicolás, San Antonio, Corralillo, San Juan Sur), Oreamuno (Cot y San Rafael), El Guarco (Comunidad el Silo), Turrialba (centro), Tucurrique, Paraíso (Centro, Purisil), 30 policías de la provincia de Cartago y docentes de las escuelas y colegios de Oreamuno y El Guarco.

En la primera etapa del proyecto se elaboraron estudios sobre el estado de la gestión de los residuos sólidos en la Municipalidad de Cartago (Quesada y Salas, 2009), los concejos municipales de distrito de Tucurrique (Torres, 2009) y Cervantes (Gaviria, 2009), las municipalidades de El Guarco (Quesada y Salas, 2009), Jiménez (Ledezma, 2009), La Unión (Gaviria, 2009), Paraíso (Ledezma, 2009) y Turrialba (Quirós, 2009). En el caso de la Municipalidad de Alvarado, los diagnósticos iniciales ya habían sido elaborados por una institución privada, por lo tanto no se hizo ninguna actualización.

A partir de estos estudios se identificaron las opciones de intervención y se seleccionaron las personas participantes en los talleres. Un resultado adicional a la capacitación anterior fue la preparación y el desarrollo del programa de Técnico en Gestión Municipal de Residuos Sólidos, en el cual se graduaron 60 personas.

Sumado a esto, se fortalecieron los centros de acopio ubicados en las municipalidades de Oreamuno y Alvarado y se trabajó en conjunto con el grupo comunal de Purisil de Paraíso. Asimismo, se recopiló información sobre la gestión de los residuos sólidos en las ocho municipalidades y dos concejos municipales de distrito de la provincia.

Además, se recopiló información de cada municipio y consejo municipal con la cual se pudo establecer un estado general de la situación de la provincia en el tema de la gestión de los residuos sólidos.

Una vez concluido este proyecto, se continuó recolectando información acerca de la gestión de los residuos sólidos en cada municipio, los sitios de disposición y los índices de indicadores de gestión, datos que se presentan en este artículo.

\section{Metodología}

El levantamiento de información se realizó mediante entrevistas de campo, consultas telefónicas, revisión de informes de la Contraloría General de la República, datos del Ministerio de Salud, análisis de sentencias judiciales y estudio de las actas municipales desde el año 2009 hasta el 201 I.

\section{Resultados}

Con base en las entrevistas y la información recopilada se logró describir las principales características de la gestión de residuos sólidos en la provincia de Cartago.

\section{Fortalezas y debilidades}

En términos generales, se encontró que para la mayoría de las municipalidades el tema de los residuos sólidos era un verdadero "dolor de cabeza" que incluía problemas de diversos tipos, incluyendo la infraestructura y el equipamiento: maquinaria vieja, obsoleta o en mal estado; problemas económicos derivados de presupuestos insuficientes, costos de tratamiento muy altos que provocaban crisis en las finanzas municipales, dificultades de recolección en sitios lejanos (Oreamuno y Turrialba, principalmente), problemas de índole social: quejas continuas de los vecinos, problemas asociados a malos olores y dificultades técnicas en el tratamiento: problemas por la mala operación de los botaderos (Cervantes, Tucurrique y Turrialba principalmente), inexistencia de programas de recolección separada (a excepción de Jiménez y Alvarado) y desconocimiento general de la gestión eficiente de los residuos sólidos. Un resumen de esto puede apreciarse en la figura I. 


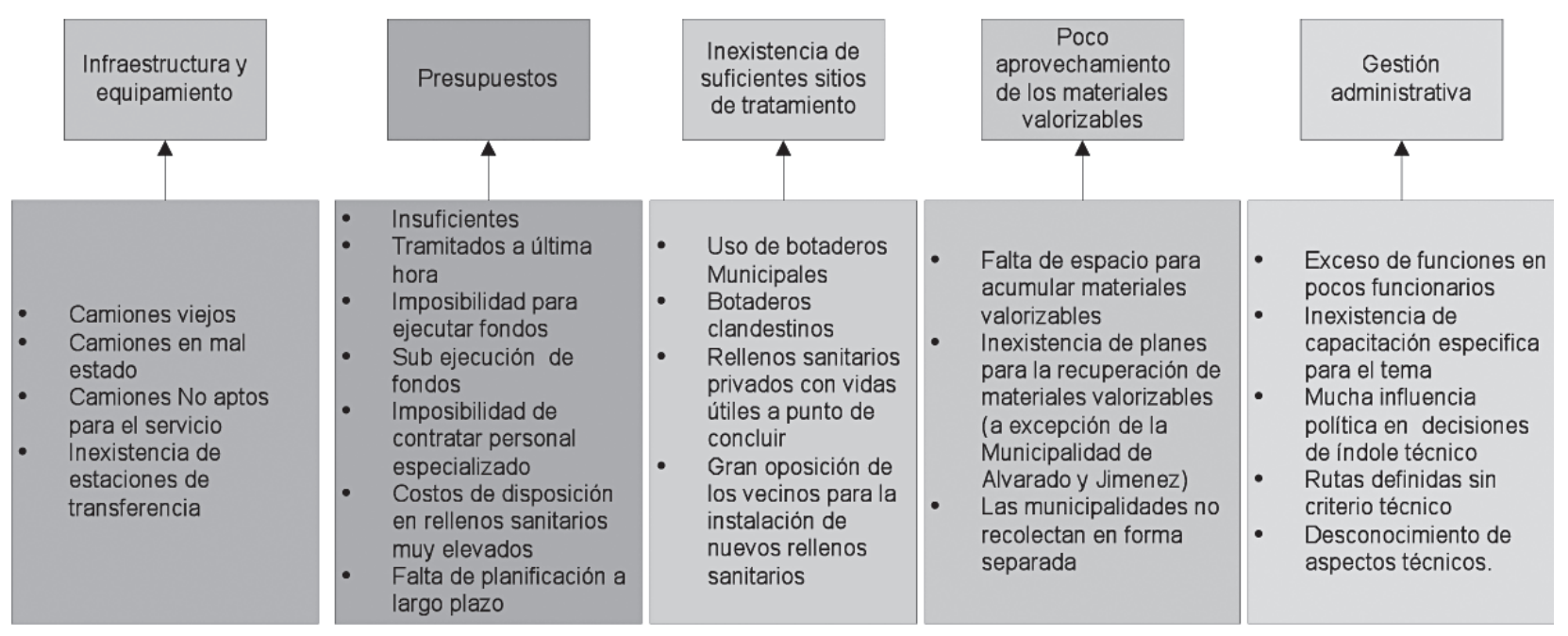

Figura I. Principales problemas detectados en la gestión de los residuos sólidos en la provincia de Cartago.

Las municipalidades de Alvarado y Jiménez mostraron una diferencia fundamental con respecto a las otras, ya que contaban con sus propios centros de recuperación de materiales, recolección separada, programas de recuperación de materiales valorizables y un compromiso ambiental y político claro y definido. La Municipalidad de Alvarado también cuenta con un programa de recuperación de envases de agroquímicos, ya que en la zona, principalmente agrícola, el uso de estos insumos es de gran importancia. Además, ya construyeron una compostera de tamaño industrial (figura 2) y están a punto de contar con un biorreactor para el tratamiento de los residuos biodegradables.

En el caso de las municipalidades de Cartago y Oreamuno, ya cuentan con estudios de composición de residuos sólidos y tienen muy adelantados sus planes de gestión integral de este tipo de desechos.

La Municipalidad de Turrialba y los concejos municipales de Cervantes y Tucurrique presentan un serio problema de disposición de los residuos, ya que utilizan botaderos municipales que son manejados en forma inapropiada.

Por otra parte, se encontraron muchas fortalezas que son comunes a la mayoría de las municipalidades, que incluyen la voluntad comunal de grupos organizados y del sector político para mejorar la gestión de los residuos sólidos, un elevado porcentaje de la población que mostraba sensibilización ante la necesidad de separar los materiales, sobre todo entre los más jóvenes; un gran número de grupos organizados que separaban los residuos valorizables y empresas recicladoras. Como puede observarse en el cuadro I, principalmente en el cantón de Cartago se encuentran importantes empresas de reciclaje de plástico, vidrio, metales, desechos electrónicos y residuos biodegradables, lo que le da una gran fortaleza a la provincia.

En términos generales, en la provincia de Cartago se evidencia una gran cantidad de actores sociales que están recolectando residuos valorizables, sumado a la presencia de empresas de reciclaje, sin embargo, el sector municipal no ha podido convertirse en el rector del proceso, a excepción de las municipalidades de Alvarado y Jiménez, que tienen avances muy importantes en el tema.

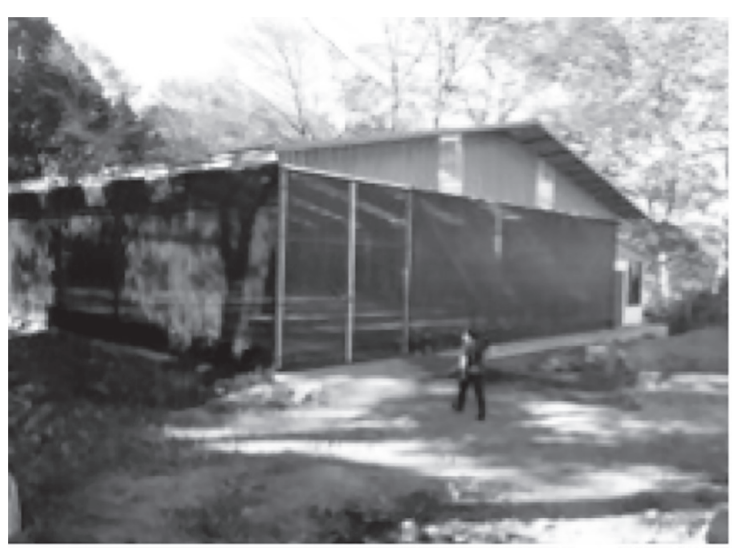

Figura 2. Compostera de desechos orgánicos municipales de la Municipalidad de Alvarado. 
Cuadro I. Principales centros de reciclaje de la provincia de Cartago.

\begin{tabular}{|c|c|c|}
\hline Nombre & Municipalidad o ubicación aproximada & Materiales que reciclan \\
\hline $\begin{array}{l}\text { Grupo Rosure (SR } \\
\text { TICOEXPORTA) }\end{array}$ & $\begin{array}{l}\text { Costado Sur del Palí, Barrio El Carmen } \\
\text { http://www.costaricareciclaje.com/esp/comercializadoras/ } \\
\text { chatarra.php, email sercliente@rosure.net }\end{array}$ & $\begin{array}{l}\text { Metales ferrosos, aluminio, cobre, } \\
\text { acero, etc. }\end{array}$ \\
\hline Abono BIOS & Ochomogo, detrás de Recope. & Abono orgánico \\
\hline INA & La Chinchilla de Cartago & Compost \\
\hline VICESA & Quircot reciclaje@grupovical.com & Vidrio \\
\hline Geocycle & Agua Caliente de Cartago & $\begin{array}{l}\text { Coproceso térmico de materiales } \\
\text { ordinarios y peligrosos. }\end{array}$ \\
\hline ITCR & Los Ángeles Cartago & $\begin{array}{l}\text { Tratamiento de desechos } \\
\text { peligrosos de laboratorios in situ. }\end{array}$ \\
\hline Mueblería San Nicolás & Taras & $\begin{array}{l}\text { Reparación y reutilización de } \\
\text { tarimas }\end{array}$ \\
\hline Celco & Ochomogo & Re-destilación de solventes \\
\hline Fundidora Díaz & Lima & $\begin{array}{l}\text { Reciclaje de aluminio, bronce y } \\
\text { cobre. }\end{array}$ \\
\hline Héctor Campos & $\begin{array}{l}\text { Agua Caliente de Cartago, } 25 \text { metros al norte del } \\
\text { cementerio http://www.cartagovirtual.com/zonaverde/ } \\
\text { index.htm }\end{array}$ & $\begin{array}{l}\text { Reutilización de papel kraft para } \\
\text { hacer encerados. }\end{array}$ \\
\hline GEEP Costa Rica. & El Guarco. www.geep.cr & $\begin{array}{l}\text { Desechos electrónicos y } \\
\text { eliminación segura de datos }\end{array}$ \\
\hline Gente reciclando & Ochomogo & Plásticos \\
\hline Reciclaje de estañones & Cartago, reciclajedeenvases.com & Envases plásticos y metálicos \\
\hline $\begin{array}{l}\text { REPLACORI, reciclados } \\
\text { plásticos de Costa Rica }\end{array}$ & Tejar, el Guarco. www.tejaplastica.com & $\begin{array}{l}\text { Plásticos de envases, cajas, bolsas, } \\
\text { bolsas bananeras. Fábrica de tejas y } \\
\text { esquineros plásticos. }\end{array}$ \\
\hline $\begin{array}{l}\text { Reciclado de envases } \\
\text { metálicos y plásticos }\end{array}$ & $\begin{array}{l}\text { San Nicolás, Cartago, } \\
\text { Email: bernyra2005@yahoo.com Sitio Web : www. } \\
\text { reciclajedeenvases.com }\end{array}$ & $\begin{array}{l}\text { Estañones metálicos y plásticos, } \\
\text { cubetas, pichingas plásticas y } \\
\text { tanquetas de } 1000 \text { litros. }\end{array}$ \\
\hline $\begin{array}{l}\text { Plycem Construsistemas } \\
\text { de Costa Rica }\end{array}$ & Cartago, 5 km hacia carretera Paraíso & Periódico \\
\hline $\begin{array}{l}\text { Energías biodegradables } \\
\text { de Costa Rica S.A }\end{array}$ & Cartago energiasbiodegradables@ice.co.cr & Aceite quemado \\
\hline Reciclaje Luna & Cartago Ilunal654@yahoo.com & Plásticos \\
\hline Fortech Química & Parque Industrial Cartago gpereira@fortech.cr & Desechos electrónicos \\
\hline
\end{tabular}

Fuentes: Consultas personales (Quesada y Salas, 2009), http://www.cartagovirtual.com/zonaverde/index.htm, Paginas Verdes de Costa Rica, http://www.redcicla.org/organizacion/afiliados.php, http://unaweb.una.ac.cr/unasostenible/ downloads/CENTROS\%20DE\%20ACOPIO\%20DE\%20MATERIAL\%20RECICLABLE. I7.pdf

\section{Estado de la recolección y el tratamiento}

la recolección de los residuos sólidos en la provincia de Cartago es brindada mayoritariamente por servicios municipales, a excepción de El Guarco y Alvarado. En las zonas urbanas, la recolección generalmente ronda el 100\%, pero en las zonas rurales la situación se invierte pues la mayoría de los habitantes entierran sus residuos sólidos biode- gradables y queman la otra parte. En el caso de la disposición, existen tres focos de contaminación en los botaderos de Turrialba (el más grande de todos), Cervantes y Tucurrique. En el cuadro 2 se muestran las particularidades de cada municipio.

Las municipalidades de la Unión, Cartago, Paraíso y Oreamuno utilizan camiones relativamente modernos (de unos 10 años) y acondicionados para el 
Cuadro 2. Resumen de la recolección de residuos sólidos en la provincia de Cartago.

\begin{tabular}{|c|c|c|c|}
\hline Cantón & Zona urbana & Zona rural & Sitio de vertido \\
\hline Turrialba & $\begin{array}{l}\text { Se atiende a } 4000 \text { habitantes de los } \\
\text { distritos Central, San Rosa, La Suiza, } \\
\text { Cabecera e Isabel. }\end{array}$ & $\begin{array}{l}\text { No reciben recolección ni } \\
\text { tratamiento }\end{array}$ & $\begin{array}{l}\text { Botadero municipal de } \\
\text { Turrialba }\end{array}$ \\
\hline Oreamuno & $\begin{array}{l}\text { Solo en los distritos de San Rafael, } \\
\text { Cot y Cipreses. }\end{array}$ & $\begin{array}{l}\text { No reciben recolección ni } \\
\text { tratamiento }\end{array}$ & Relleno sanitario Los Pinos \\
\hline Cartago & $100 \%$ & $100 \%$ & Relleno sanitario Los Pinos \\
\hline Tucurrique & $90 \%$ & $\begin{array}{l}\text { No reciben recolección ni } \\
\text { tratamiento }\end{array}$ & $\begin{array}{l}\text { Botadero municipal de } \\
\text { Tucurrique }\end{array}$ \\
\hline Cervantes & $100 \%$ & Sin datos & $\begin{array}{l}\text { Botadero municipal de } \\
\text { Cervantes }\end{array}$ \\
\hline El Guarco & $\begin{array}{l}\text { Tejar, Tobosi y el centro de San } \\
\text { Isidro }\end{array}$ & $\begin{array}{l}\text { No reciben recolección ni } \\
\text { tratamiento. Los vecinos queman la } \\
\text { basura y en las escuelas rurales la } \\
\text { entierran. }\end{array}$ & Relleno sanitario Los Pinos \\
\hline Jiménez & $100 \%$ en el distrito de Juan Viñas & No se tienen datos & $\begin{array}{l}\text { Botadero municipal de } \\
\text { Turrialba }\end{array}$ \\
\hline Alvarado & $100 \%$ en el distrito Central & No se tienen datos & Relleno sanitario Los Pinos \\
\hline La Unión & $100 \%$ & $100 \%$ & $\begin{array}{l}\text { Relleno sanitario Los Mangos, } \\
\text { en Alajuela. }\end{array}$ \\
\hline Paraíso & $90 \%$ & $\begin{array}{l}\text { Sin atención a los Altos de Araya, } \\
\text { Purisil y La Mesita, pues ellos } \\
\text { mismos los manejan para evitar el } \\
\text { cobro municipal. }\end{array}$ & Relleno sanitario Los Pinos \\
\hline
\end{tabular}

Fuente: Municipalidad de Turrialba, Intendente Municipal de Cervantes, Encargado de Residuos Sólidos de Oreamuno (Gaviria, 2009), (Quesada y Salas, 2009), consultas personales y visitas.

transporte de residuos sólidos, sin embargo, en el caso de los municipios más pequeños y alejados, los camiones tienen más de veinte años y no son aptos para el transporte de estos desechos, como puede verse en las figuras 3 y 4.

El principal tipo de tratamiento que se le da a los residuos sólidos en la provincia de Cartago es mediante el vertido, ya sea en botaderos o rellenos sanitarios. En el caso de la municipalidad de Turrialba y el concejo municipal de Tucurrique, existen órdenes de cierre pendientes desde hace varios años, sin embargo, debido a los costos, las distancias y las dificultades técnicas, ambos sitios siguen operando con los consiguientes problemas sanitarios, dado que su cierre acarrearía mayores dificultades.

\section{Estado de los centros de acopio}

En la provincia funcionan muchos centros de acopio en escuelas, colegios, centros sociales y grupos comunales, la mayoría son improvisados, no tienen licencia de funcionamiento y sobreviven marginalmente. Muchos de estos centros responden a iniciativas personales, comunales o de algún dirigente, muchas veces dejan de funcionar cuando el dirigente decide no continuar. En las figuras 5 y 6 se muestran algunos centros de acopio y se puede evidenciar su funcionamiento. Estos sitios pueden convertirse en verdaderos problemas ya sea por acumulación de roedores o como fuente de incendios. 

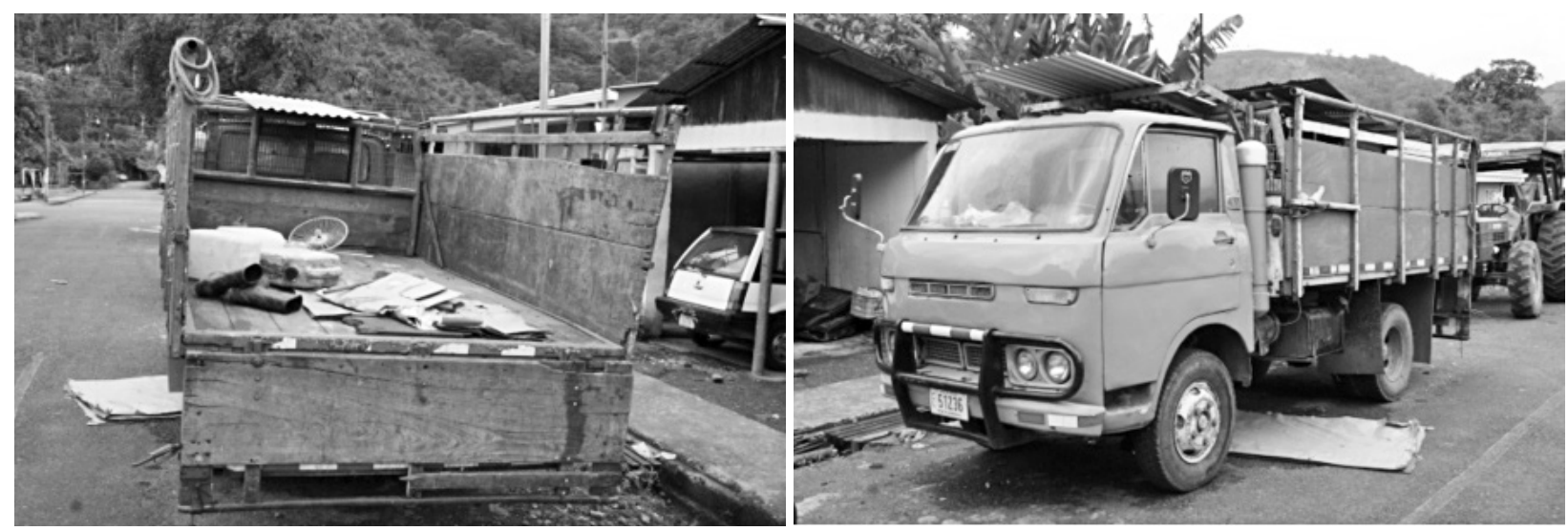

Figura 3. Estado de los camiones en Tucurrique. Fuente:Torres, 2009.
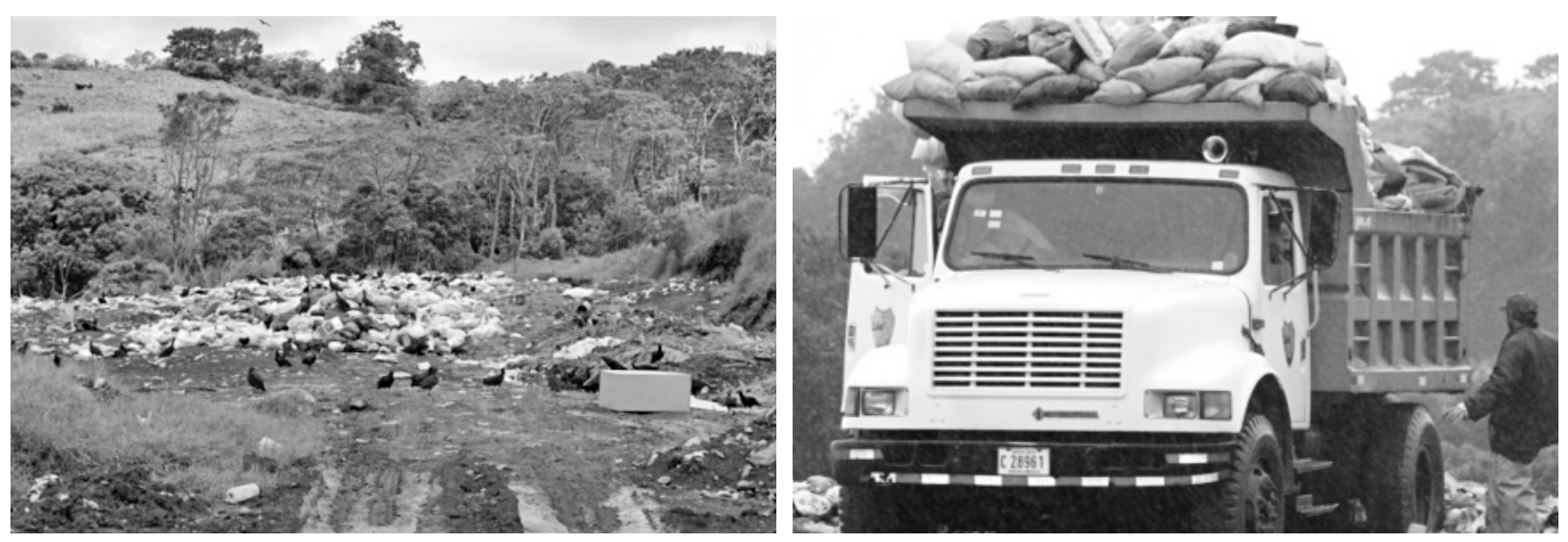

Figura 4. Fotografías del botadero en Cervantes y de la forma en que se transporta la basura. Fuente: Gaviria, 2009.

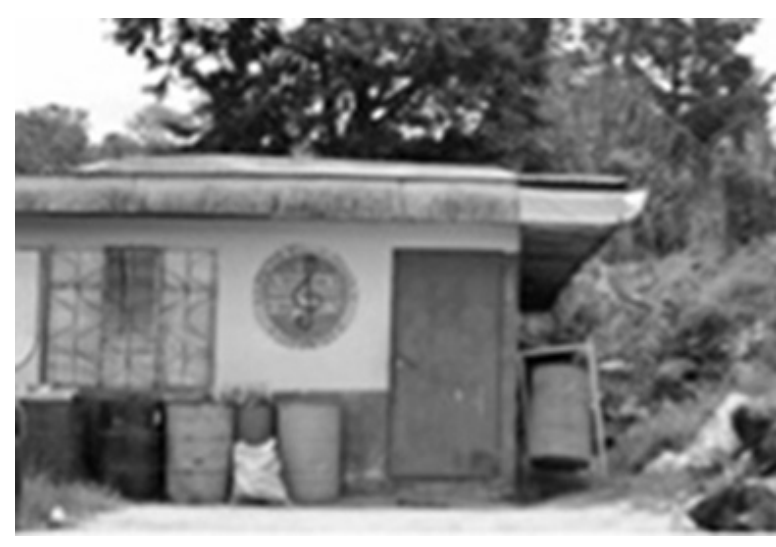

Figura 5. Centro de acopio en la Escuela de Música de Cervantes. Fuente: Gaviria, 2009.

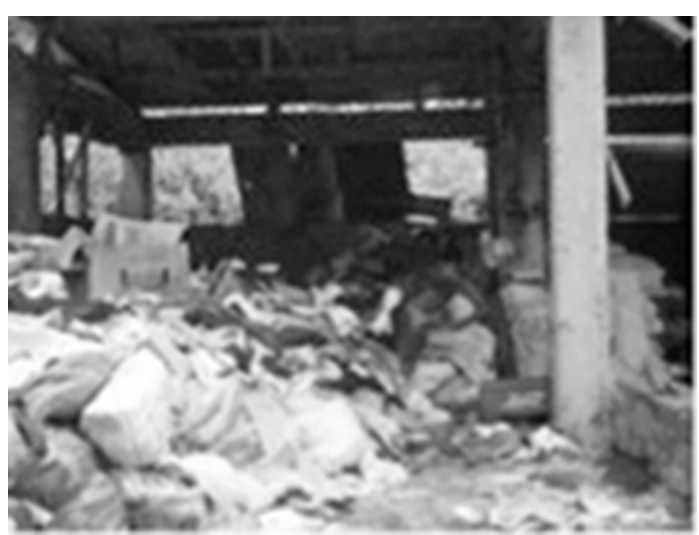

Figura 6. Interior de un centro de acopio en Tucurrique. Fuente: Torres, 2009. 
Las personas que están a cargo de los centros de acopio muchas veces no tienen conocimiento del tema y trabajan con mucho entusiasmo, pero esto no necesariamente se traduce en éxitos.

En la Municipalidad de Jiménez funciona un centro de recuperación de materiales que es un ejemplo para la provincia. En la figura 7 se observa un achiche diseñado para divulgar el programa de recuperación

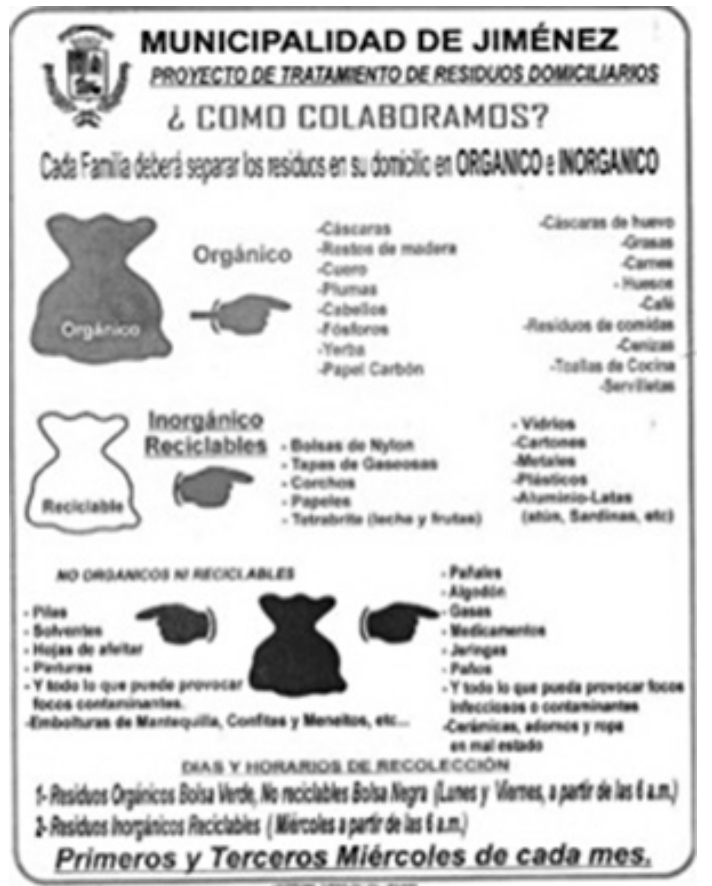

Figura 7. Afiche publicitario Municipalidad de Jiménez. Fuente: Municipalidad de Jiménez.

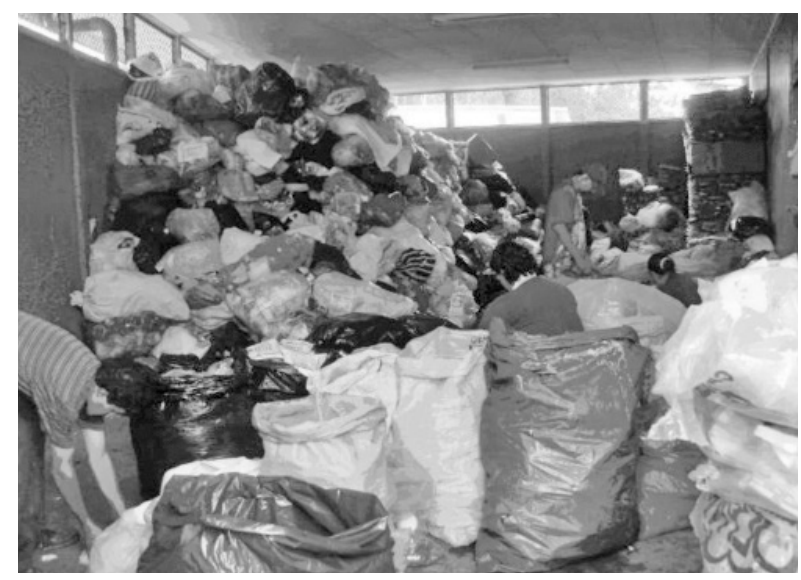

A de materiales, y en la figura 8 se muestra su interior. También existen centros de acopio municipales en Alvarado y Oreamuno.

\section{Aplicación de la Ley Gir 8839}

En el año 2009 se realizaban acciones aisladas en la provincia para gestionar adecuadamente los residuos sólidos. La más conocida y exitosa era el centro de acopio de la Municipalidad de Jiménez, junto con las políticas municipales que obligaban a los vecinos a realizar la separación en la fuente. La aplicación de estas medidas provocó enfrentamientos de los vecinos con el alcalde, e incluso en algunas ocasiones ciudadanos molestos dejaron sus bolsas con basura en la puerta del funcionario como medida de protesta por obligarlos a separarla.

Por su parte, en la Municipalidad de Alvarado, pese a contar con una situación económica muy adversa, se observó un repunte originado en parte en la intervención oportuna de sus dirigentes políticos y la participación activa de la comunidad. A inicios de 2009 se comenzó a trabajar fuertemente con el programa CYMA del gobierno alemán, de forma que esta municipalidad pudo contar con estudios diagnósticos de sus cantón. Posteriormente, lograron dirigir fondos para la capacitación de personal y diseñaron un centro de acopio municipal que era operado en forma voluntaria por miembros de la comunidad y luego fue posible pagar un subsidio a vecinos de escasos recursos por esta labor. En 2010 decidieron implementar una oficina de ges-

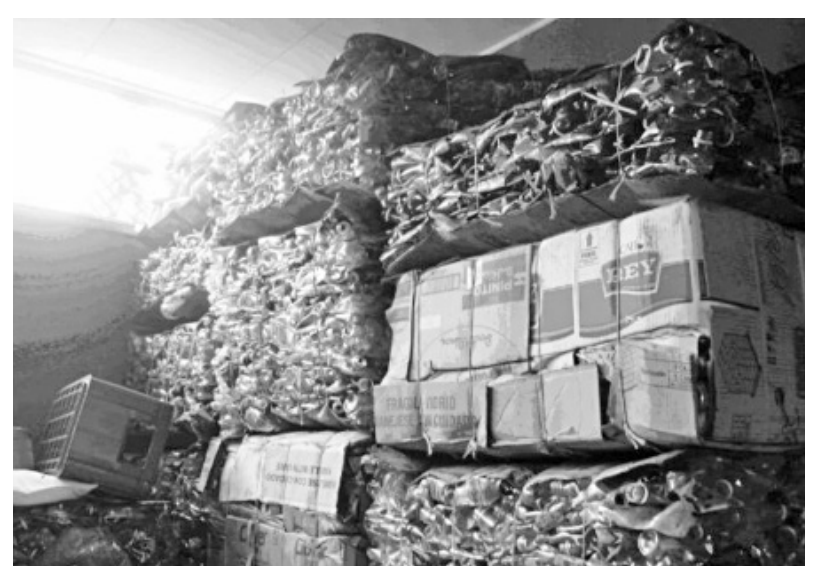

B

Figura 8. Interior del centro de acopio de la Municipalidad de Jiménez. En la fotografía A se muestra la recolección inicial antes de la separación y en la fotografía B el material ya procesado y listo para su venta. Fuente: Ledezma, 2009. 
tión ambiental y se contrataron empleados; hasta la fecha, están realizando una labor destacable en la provincia.

De igual forma, las municipalidades de Cartago, Paraíso, La Unión y Turrialba apostaron por contar con oficinas de gestión ambiental para atender los problemas derivados de los residuos sólidos.

A raíz de la aprobación de la Ley GIR (8839) en 2010 , se obligó a todas las municipalidades a contar con oficinas de gestión ambiental, sin embargo, se está a la espera del reglamento específico que definirá el plazo definitivo para que esto ocurra. Esta ley también propone, entre otras cosas, diseñar planes de manejo de residuos sólidos, elaborar estudios de composición de materiales y contar con reglamentos específicos para una gestión integral.

Entre los municipios se observaron diferencias importantes en la conformación de los concejos municipales, los alcaldes y el personal.

Llamó fuertemente la atención que ciertos municipios, a pesar de contar con personal capacitado, recursos y buenas ideas, comunidades organizadas y demás aspectos favorables, no pudieron avanzar cuando el concejo municipal estaba polarizado y se desgastaba en discusiones inútiles o buscando culpables.

También se observó una gran desmotivación de los funcionarios municipales en ambientes altamente polarizados, o bien con estructuras de gestión del gasto ineficientes. Por ejemplo, se hacían asignaciones de presupuesto al filo del tiempo de ejecución, dejándole al funcionario municipal poca o ninguna posibilidad de ejecución financiera. También en algunas municipalidades se observó el doble recargo de funciones con respecto al manejo de los acueductos y los problemas de residuos sólidos.

Con el fin de evaluar la gestión municipal de los residuos sólidos, durante 2012 se entrevistó, mediante consultas telefónicas y correos electrónicos, a los encargados municipales sobre el grado de atención a estos temas. Como puede verse en el Cuadro 3, la mitad de las municipalidades de la provincia ya contaban con oficinas de gestión ambiental, lo cual fue una buena noticia, sin embargo, en los temas de estudios de composición y demás aspectos requeridos por la ley, el avance ha sido lento.

Para comparar el avance de las municipalidades, en este artículo se propone una calificación compuesta por algunos de los requisitos solicitados por la ley.
Es importante aclarar que esta calificación es preliminar, pues es necesario considerar otros aspectos, tales como presupuestos de operación, ejecución de presupuestos, capacitación de los funcionarios municipales o evaluación de los sitios de disposición, que a la fecha no han sido medidos, por lo cual se decidió diseñar esta clasificación de forma preliminar.

La calificación de cada municipio consiste en una combinación de: I) la capacidad económica para atender los problemas, que en este caso se evaluará con la existencia o no de la oficina ambiental con presupuesto, 2) las acciones de planificación para cumplir con la ley $\mathrm{GIR}$, en que se utilizará como indicador la existencia de planes de manejo de residuos sólidos y la existencia de datos de estudios de composición, 3) las acciones concretas para aplicar la recolección discriminada y valorizar los residuos sólidos, considerando para este ítem el contar con la recolección separada y reglamentos específicos que incluyan la obligatoriedad de la recolección discriminada; y finalmente 4) sitios de tratamiento adecuados, para lo cual solamente se consideró el uso de rellenos sanitarios o botaderos como criterio de calificación.

La fórmula utilizada es la siguiente:

Calificación = Capacidad económica + Acciones de planificación + Recolección discriminada + Sitios de vertido.

Considerando que cada aspecto tiene una participación equitativa, a cada ítem se le asignaría un 25\% de participación en la calificación final. Los resultados se muestran en el cuadro 3.

\section{Conclusiones}

La implementación de la ley GIR en la provincia avanza según el ritmo de cada municipio; algunos muestran avances importantes y otros más bien retrocedieron. Es claro que la aplicación de la ley ayuda a mejorar la situación pero no es suficiente ya que existen aspectos imposibles de reglamentar.

Analizando los municipios exitosos, se identificaron al menos cinco fortalezas comunes y que deben existir simultáneamente para que las acciones Ileguen a buen término, a saber:

I. Concejos municipales que privilegian los intereses comunales antes que los partidarios.

2. Alcaldes municipales proactivos comprometidos con el tema. 


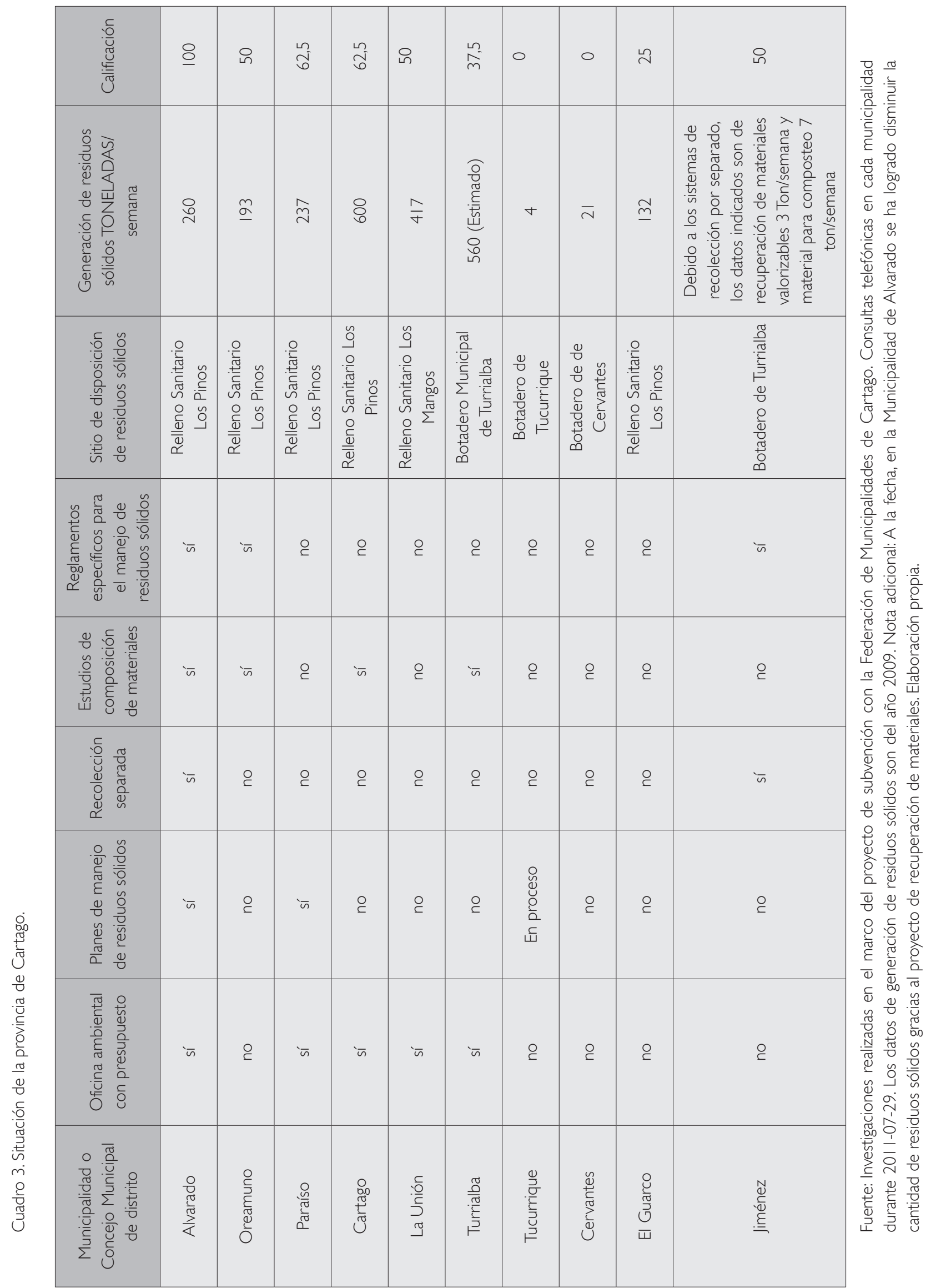


3. Existencia de una oficina de gestión ambiental con suficiente presupuesto.

4. Respeto por las actividades realizadas por los profesionales responsables de las oficinas de gestión ambiental.

5. Participación activa de la comunidad

Cuando existían estas condiciones, ni siquiera era necesario que existiera la ley para poner en marcha acciones concretas en pro de la gestión adecuada de los residuos sólidos.

Por esta razón, consideramos que es urgente la capacitación del sector político en temas de toma de decisiones, conceptos ambientales, ética, responsabilidad social, empatía y priorización de actividades.

También resulta obvia la necesidad de contar con la oficina de gestión ambiental, que no ha querido implementarse en algunos municipios argumentando falta de recursos o bien que se ha implementado a medias, utilizando como mecanismo una comisión ambiental, que no tiene los recursos ni el tiempo para ejecutar acciones.

Además de las cinco acciones anteriores, para avanzar en el proceso de la gestión integral de los residuos sólidos es necesario profesionalizar el sector municipal. Para esto se deben contratar ingenieros ambientales que conozcan los aspectos ambientales, el diseño de rutas, los diseños de tratamiento, los costos involucrados, los impactos ambientales, así como las estructuras de costos e inversión.

También es necesario mejorar las condiciones de trabajo de los recolectores así como su autoestima y la percepción que tiene la comunidad de su trabajo, ya que estos trabajadores son parte fundamental del servicio sanitario que presta la municipalidad. Es necesario aumentar su escolaridad, para que puedan ayudar en la valorización de los residuos que ingresan a los centros de tratamiento, convirtiéndose en educadores de los vecinos a los cuales atienden.

Todo esto incide en el aprovechamiento de los residuos sólidos de la provincia, que ascienden a cerca de 97,000 toneladas anuales.

La buena gestión de los residuos sólidos implica invertir las cantidades óptimas del presupuesto municipal de manera que no afecte la inversión en otros aspectos ambientales de importancia, tanto sanitaria como de protección. Las municipalidades no deben descuidar los sitios de recarga, los bos- ques, el asfaltado municipal, la limpieza de parques, el embellecimiento de la ciudad, las oportunidades para la mujer, etc.

El manejo de los residuos sólidos en la provincia de Cartago requiere la atención urgente por parte de los tomadores de decisión para aprovechar los residuos valorizables, considerando que la provincia cuenta con empresas de reciclaje, que incluso reciben materiales de todo Centroamérica (VICESA y GEOCYCLE).

Estas acciones pueden ser implementadas y la prueba de ello es que dos de los municipios más pequeños y con menos recursos han logrado posicionarse como ejemplos a nivel nacional. En el caso de la Municipalidad de Jiménez, desde hace más de 10 años viene ejecutando acciones para recuperar los materiales valorizables y educar a su población. Por su parte, la Municipalidad de Alvarado ha invertido en obras de infraestructura para darle tratamiento a la parte biodegradable de sus residuos sólidos, además, cuenta con una oficina de gestión ambiental altamente calificada y muy motivada.

En estos momentos, la prioridad consiste en que las municipalidades puedan implementar planes concretos de recolección de residuos valorizables. La población ya ha sido sensibilizada y solo espera contar con espacios para poder colocar los materiales.

\section{Bibliografía}

Gaviria, L. (2009). Diagnóstico inicial de la situación de los residuos sólidos en la Municipalidad de Cervantes. Cartago: ITCR.

Gaviria, L. (2009). Diagnóstico inicial de la situación de los residuos sólidos en la Municipalidad de Oreamuno. Cartago: ITCR.

Ledezma, A. (2009). Diagnóstico inicial de la situación de los residuos sólidos en la Municipalidad de Jiménez. Cartago: ITCR.

Ledezma, A. (2009). Diagnóstico inicial de la situación de los residuos sólidos en la Municipalidad de Paraíso. Cartago: ITCR.

Ledezma, A. (2009). Diagnóstico inicial de la situación de los residuos sólidos en la Municipalidad de Paraíso. Cartago: ITCR.

Quesada, H. \& Salas, J. (2009). Diagnóstico inicial de la situación de los residuos sólidos en la Municipalidad de El Guarco. Cartago: ITCR.

Quesada, H. \& Salas, J. (2009). Diagnóstico inicial de la situación de los residuos sólidos en la Municipalidad de Cartago. Cartago: ITCR.

Quirós, N. (2009). Diagnóstico inicial de la situación de los residuos sólidos en la Municipalidad de Turrialba. Cartago: ITCR.

Torres, R. (2009). Diagnóstico inicial de la situación de los residuos sólidos en la Municipalidad de Tucurrique. Cartago: ITCR. 